KYUNGPOOK Math. J. 55(2015), 773-777

http://dx.doi.org/10.5666/KMJ.2015.55.4.773

pISSN 1225-6951 eISSN 0454-8124

(c) Kyungpook Mathematical Journal

\title{
A Fixed Point on Generalised Cone Metric Spaces
}

\author{
Sudip Kumar PAL* \\ Department of Mathematics, University of Kalyani, Kalyani-741-235, India \\ e-mail : sudipkmpal@yahoo.co.in \\ MANOJIT MAITY \\ Assistant Teacher in Mathematics, Boral High School, Kolkata-700-154, India \\ e-mail : mepsilon@gmail.com
}

Abstract. The aim of this paper is to prove a fixed point theorem on a generalised cone metric spaces for maps satisfying general contractive type conditions.

\section{Introduction}

The study of fixed points of mappings satisfying certain contractive conditions has been very active area of research. Recently Long-Guang and Xian [9] generalised the concept of a metric space, by introducing cone metric spaces, and obtained some fixed point theorem for mappings satisfying certain contractive conditions. One can consider a generalisation of a cone metric space by replacing the triangle inequality by a more general inequality. As such, every cone metric is a generalised cone metric space but the converse is not true. However the interesting point to note that two very important fixed point theorems, namely Banach's fixed point theorem and Ciric's fixed point theorem have already establised in such a space. In this paper we continue in this direction and prove a fixed point theorem of Boyed and Wang [2],[5] under fairly general condition a generalised cone metric spaces.

\section{Section 2}

Let $E$ be a real Banach space. A nonempty convex closed subset $P \subset E$ is called a cone in $E$ if it satisfies:

* Corresponding Author.

Received September 13, 2013; accepted April 11, 2014.

2010 Mathematics Subject Classification: 54C60, 54H25.

Key words and phrases: Generalised cone metric space, contractive mapping, fixed point. The research of the First Author was funded by University Grants Commission, Govt. of India through the D. S. Kothari Post Doctoral Fellowship. 
(i) $P$ is closed, nonempty and $P \neq\{0\}$,

(ii) $a, b \in \mathbb{R}, a, b \geq 0$ and $x, y \in P$ imply that $a x+b y \in P$,

(iii) $x \in P$ and $-x \in P$ imply that $x=0$.

The space $E$ can be partially ordered by the cone $P \subset E$; i. e. $x \leq y$ if and only if $y-x \in P$. Also we write $x<<y$ if $y-x \in$ int $P$, where int $P$ denotes the interior of $P$.

A cone $P$ is called normal if there exists a constant $K>0$ such that $0 \leq x \leq y$ implies $\|x\| \leq K\|y\|$.

In the following we always suppose that $E$ is a real Banach space, $P$ is a cone in $E$ and $\leq$ is partial ordering with respect to $P$.

Definition 2.1. Let $X$ be a nonempty set and let $E$ be a Banach space with cone $P$ and $d: X^{2} \rightarrow E$ be a mapping such that for all $x, y \in X$ and for any $k(k \geq 2)$ distinct points $z_{1}, z_{2}, \ldots \ldots, z_{k}$ in $X$ each of them different from $\mathrm{x}$ and $\mathrm{y}$, one has

1. $\theta \leq d(x, y)$ for all $x, y \in X$, and $d(x, y)=\theta$ if and only if $x=y$.

2. $d(x, y)=d(y, x)$ for all $x, y \in X$

3. $d(x, y) \leq d\left(x, z_{1}\right)+d\left(z_{1}, z_{2}\right)+\ldots . .+d\left(z_{k}, y\right)$ for all $x, y, z_{1}, z_{2}, \ldots \ldots, z_{k}$ in $X$. i.e. $\left\{d\left(x, z_{1}\right)+d\left(z_{1}, z_{2}\right)+\ldots . .+d\left(z_{k}, y\right)-d(x, y)\right\} \in P$

Then we say $(X, d)$ is a generalised cone metric space (or shortly g.c.m.s.).

Throughout this section a g.c.m.s. will be denoted by $(X, d)$ (or sometimes by $X$ only) and $\mathbb{N}$ denote the set of all naturals.

Any cone metric space is a g.c.m.s. but the converse is not true [1]. We first recall some basic definitions.

Definition 2.2. A sequence $\left\{x_{n}\right\}_{n \in \mathbb{N}} \in X$ is said to be a g.c.m.s. convergent if for every $\varepsilon$ in $E$ with $\theta<\varepsilon$, there is an $N \in \mathbb{N}$ such that for all $n>N, \varepsilon-d\left(x_{n}, x\right) \in P$ for some fixed $x \in X$.

Definition 2.3. A sequence $\left\{x_{n}\right\}_{n \in \mathbb{N}} \in X$, is said to be a g.c.m.s. Cauchy sequence if for every $\varepsilon \in E$ with $\theta<\varepsilon$, there is an $N \in \mathbb{N}$ such that for all $m, n>N$, $\varepsilon-d\left(x_{n}, x_{m}\right) \in P$. in $X$.

We say that a g.c.m.s is complete if every Cauchy sequence in $X$ is convergent

Definition 2.4. A mapping $T: X \rightarrow X$ is said to be contractive if for any two points $x, y \in X, \quad d(x, y)-d(T x, T y) \in P$.

Definition 2.5. A function $f: E \rightarrow P$ is said to be upper semicontinuous at $x_{0} \in E$ if there exists a neighbourhood $U$ of $x_{0}$ such that $f(x)+\epsilon \in P$, for all $x \in U$.

We now prove the following fixed point theorem for Boyd and Wong's contractive mappings [2], [5]. 
Theorem 2.6. Let $X$ be a complete g.c.m.s. and let $T: X \rightarrow X$ satisfies

$$
\psi(d(x, y))-d(T x, T y) \in P,
$$

where $\psi: \bar{P} \rightarrow E$ is upper semicontinuous from right on $\bar{P}$ (the closure of the range d) and satisfies $\psi(t)<t$ for all $t \in \bar{P}-\{0\}$. Then $T$ has a unique fixed point $x_{0}$ and $T^{n} x \rightarrow x_{0}$ for all $x \in X$.

Proof. Given $x \in X$, define

$$
c_{n}=d\left(T^{n} x, T^{n-1} x\right) .
$$

since $d(T x, T y) \leq \psi(d(x, y))<d(x, y)$, the sequence $\left\{c_{n}\right\}_{n \in \mathbb{N}}$ ie decreasing. Suppose $c_{n} \rightarrow c \in E$. Then if $c>0$, we have $\psi\left(c_{n}\right)-c_{n}+1 \in P$. Then $\limsup _{t \rightarrow c^{+}} \psi(t)-c \in P$. i.e. $\psi(t)-c \in P$ which is a contradiction. Therefore $c_{n} \rightarrow 0$.

For each $x \in X$, consider the sequence $\left\{T^{n} x\right\}$. First assume that it is eventually constant. So there is some $n \in \mathbb{N}$ such that $T^{m} x=T^{n} x=y$ for each $m>n$.

Then $T^{m-n}\left(T^{n} x\right)=T^{n} x$, so denoting $k=m-n$, we have $T^{k} y=y$ for all $k \in \mathbb{N}$. It follows that $d(y, T y)=d\left(T^{k} y, T^{k+1} y\right)=c_{k}$ for all $\mathrm{k}$, and since $c_{k} \rightarrow 0$, $d(y, T y)=0$, so $y=T y$. Then $\mathrm{y}$ is a fixed point of $\mathrm{T}$.

If $\left\{T^{n} x\right\}$ is not eventually constant, then it has a subsequence with pairwise distinct terms. Without loss of generality, assume that $\left\{T^{n} x\right\}$ is this subsequence. We shall show that $\left\{T^{n} x\right\}$ is a g.c.m.s. Cauchy sequence. By contradiction suppose that there is an $\varepsilon>0$ and sequences $\left\{m_{k}\right\},\left\{n_{k}\right\}$ of positive integers with $k \leq n_{k}<$ $m_{k}$ such that

$$
\varepsilon-d\left(T^{m_{k}} x, T^{n_{k}} x\right) \notin P \text { for all } k \in \mathbb{N} .
$$

Since this is true for all $k \in \mathbb{N}$, we can conclude that for all $k \in \mathbb{N}$, there will exist $n_{k} \geq k$ and an infinite number of $m_{k}>n_{k}$ for which

$$
\frac{\varepsilon}{3}-d\left(T^{m_{k}} x, T^{n_{k}} x\right) \in P .
$$

For otherwise let $m_{1}>n(K)$ be the highest positive integer for which (2.4) holds. Since $c_{k} \rightarrow 0$ as $k \rightarrow \infty$ we can find $m_{2} \in \mathbb{N}$ such that

$$
c_{k}=\frac{\varepsilon}{3}-d\left(T^{k} x, T^{k-1} x\right) \in P \text { for all } k \geq m_{2} .
$$

Now if $m_{0}=\max \left(m_{1}, m_{2}\right)$ then for any $i, j>m_{0}$ $\frac{\varepsilon}{3}-d\left(T^{i} x, T^{i+1} x\right) \in P \Rightarrow \frac{\varepsilon}{3}-d\left(T^{i} x, T^{j} x\right) \in P$, if $j=i+1 \Rightarrow \varepsilon-d\left(T^{i} x, T^{j} x\right) \in$ $P \Rightarrow \frac{\varepsilon}{3}-d\left(T^{i} x, T^{i+1} x\right) \in P ; \frac{\varepsilon}{3}-d\left(T^{i+1} x, T^{n} x\right) \in P ; \frac{\varepsilon}{3}-d\left(T^{n} x, T^{j} x\right) \in P \Rightarrow$ $\varepsilon-d\left(T^{i} x, T^{j} x\right) \in P$ if $j>i+1$, which contradicts (2.3). 
Now in the view of (2.4) we can choose $m_{k}$ as the least positive integer greater than $n_{k}+2$ for which

$$
d / 3=\frac{\varepsilon}{3}-d\left(T^{m_{k}} x, T^{n_{k}} x\right) \in P \text { for all } k \in \mathbb{N} .
$$

Assume that $k \geq m_{2}$. Now if

(i) $m \geq n+5$ then clearly, $c_{m}-d\left(T^{m} x, T^{m+1} x\right) \in P ; c_{m-1}-d\left(T^{m-1} x, T^{m-2} x\right) \in$ $P ; \frac{\varepsilon}{3}-d\left(T^{m-1}, T^{n} x\right) \in P$. This implies $\left(c_{m}+c_{m-1}+\frac{\varepsilon}{3}-d\left(T^{m} x, T^{n} x\right) \in P\right.$ i.e. $\left(2 c_{k}+\frac{\varepsilon}{3}-d\left(T^{m} x, T^{n} x\right) \in P\right.$.

Choose $d_{k}=\left(2 c_{k}+\frac{\varepsilon}{3}-d\left(T^{m} x, T^{n} x\right) \in P\right.$.

(ii) If $m=n+3$ then by (2.5)

$\frac{\varepsilon}{3}-d\left(T^{m-2} x, T^{n} x\right) \in P$, so $c_{k}-d\left(T^{m} x, T^{m-1} x\right) \in P ; c_{k}-d\left(T^{m-1} x, T^{m-2} x\right) \in$ $\stackrel{3}{P}$ and $\frac{\varepsilon}{3}-d\left(T^{m-2} x, T^{n} x\right) \in P$

implies $\left(2 c_{k}+\varepsilon / 3\right)-d\left(T^{m} x, T^{n} x\right)=d_{k} \in P$.

(iii) If $m=n+4$ then $c_{k}-d\left(T^{n} x, T^{n+1} x\right) \in P$; $c_{k}-d\left(T^{n+1} x, T^{n+2} x\right) \in P$; $c_{k}-d\left(T^{n+2} x, T^{n+3} x\right) \in P ; \frac{\varepsilon}{3}-d\left(T^{n+3} x, T^{n+4} x\right) \in P$. This implies $\left(3 c_{k}+\right.$ $\left.\frac{\varepsilon}{3}\right)-d\left(T^{m} x, T^{n} x\right)=d_{k} \in P$.

Hence $\frac{\varepsilon}{3}-d_{k} \in P$ as $k \rightarrow \infty$.

Again, $c_{k}-d\left(T^{m} x, T^{m+1} x\right) \in P ; c_{k}-d\left(T^{m+1} x, T^{n+1} x\right) \in P$; i.e.

$\psi\left(d\left(T^{m} x, T^{n} x\right)\right)-d\left(T^{n+1} x, T^{n} x\right) \in P$.

Hence $\psi\left(d_{k}\right)-d\left(T^{n+1} x, T^{n} x\right) \in P$. Which shows that

$$
2 c_{k}+\psi\left(d_{k}\right)-d\left(T^{m} x, T^{n} x\right)=d_{k} \in P
$$

Thus as $k \rightarrow \infty$ from (2.7), we obtain $\psi\left(\frac{\varepsilon}{3}\right)-\frac{\varepsilon}{3} \in P$,

which contradicts the given condition since $\varepsilon>0$.

Therefore in this case $\left\{T^{n} x\right\}$ is a g.c.m.s. Cauchy and as $X$ is complete, $\left\{T^{n} x\right\}$ converges to a point $x_{0}$ in $X$.

We shall show that $T x_{0}=x_{0}$. We divide the proof into two parts. First let $T^{n} x$ be different from both $x_{0}$ and $T x_{0}$ for any $n \in \mathbb{N}$. Then

$d\left(T x_{0}, T^{n} x\right)+d\left(T^{n} x, T^{n+1} x\right)+d\left(T^{n+1} x, T x_{0}\right)-d\left(x_{0}, T x_{0}\right) \in P$

i.e. $d\left(x_{0}, T^{n} x\right)+c_{n+1}+\psi\left(d\left(x_{0}, T^{n} x_{0}\right)\right)-d\left(x_{0}, T x_{0}\right) \in P$

hence $d\left(x_{0}, T x_{0}\right)+c_{n+1}+d\left(x_{0}, T^{n} x_{0}\right)-d\left(x_{0}, T x_{0}\right) \in P$. Which gives

$\varepsilon-d\left(x_{0}, T x_{0}\right) \in P$ for any $\varepsilon>0$ and as $n \rightarrow \infty$.

Which implies $T x_{0}=x_{0}$.

Next assume that $T^{k} x=x_{0}$ or $T^{k} x=T x_{0}$ for some $k \in \mathbb{N}$.

Obviously then $x_{0} \neq x$ and one can easily show that $\left\{T^{n} x_{0}\right\}$ is a sequence with the following properties.

(i) $\varepsilon-\lim _{n \rightarrow \infty} d\left(T^{n} x_{0}, x_{0}\right) \in P$.

(ii) $x_{0}-T^{n} x_{0} \notin P$ for any $n \in \mathbb{N}$.

(iii) $T^{r} x_{0}-T^{p} x_{0} \notin P$ for any $p, r \in \mathbb{N}, p \neq r$. 
Hence proceeding the above it immediately follows that $x_{0}$ is a fixed point of $T$. That the fixed point of $T$ is unique easily follows from the definition of $T$.

\section{References}

[1] A. Branciari, A fixed point theorem of Banach-Caccioppoli type on a class of generalised metric spaces, Publ. Math. Debrecen, 57(2000), 31-37.

[2] D. W. Boyd and J. S. W. Wong, On nonlinear contraction, Proc. Amer. Math. Soc., 20(1996), 458-464.

[3] L. J. B. Ćirić, A generalization of Banach's contraction principle, Proc. Amer. Math. Soc., 45(1974), 267-273.

[4] P. Das, A fixed point theorem on a class of generalized metric spaces, Korean J. Math. Sci., 9(2002), 29-33.

[5] P. Das and L. K. Dey, Fixed point of contractive mappings in generalized metric spaces, Math. Slovaca, 59(4)(2009), 499-504.

[6] M. Edelstein, On fixed point and periodic points under contraction mappings, J. London Math. Soc., 37(2)(1962), 74-79.

[7] D. Ilić and V. Rakočević, Common fixed points for maps on cone metric space, J. Math. Anal. Appl., 341(2008), 876-882.

[8] B. K. Lahiri and P. Das, Fixed point of a Ljubomir Ćirić's quasi-contraction mapping in a generalized metric space, Publ. Math. Debrecen 61(2002), 589-594.

[9] H. Long-Guang and Z. Xian, Cone metric spaces and fixed point theorems of contractive mapping, J. Math. Anal. Appl. 322(2007), 1468-1476.

[10] J. O. Olaeru and H. Akewe, An extension of Gregus fixed point theorem, Fixed Point Theory Appl., (2007), Article ID 657914.

[11] E. Rakotch, A note on contractive mappings, Proc. Amer. Math. Soc. 13(1962), 459465.

[12] S. Reich and A. J. Zaslavski, Almost all non-expansive mappings are contractive, C. R. Math. Acad. Sci. Soc. R. Can., 22(2000), 118-124.

[13] S. Reich and A. J. Zaslavski, The set of non contractive mappings is $\sigma$-porous in the space of all non-expansive mappings, C. R. Math. Acad. Sci. Paris, 333(2001), 539-544. 